\title{
The Conceptual Design of a Smart Wrist Orthosis and Functional Performance - Project Overview
}

\author{
Zoran Domitran*, Robert Mašović, Jure Serdar, Mislav Jelić
}

\begin{abstract}
The idea of the project was to find a conceptual design of a multi-functional smart orthosis for wrist fixation. The application of additive technologies presents the potential in designing a customised orthosis for every patient individually. By using pre-generated $3 \mathrm{~d}$ models of various hand shapes it is possible to prepare models for several shapes and sizes of forearms and hands. The conceptual design provides a possible solution for a two-part orthosis bound around the forearm and a modular extension to stabilize the wrist without additional compression. The multi-functionality occurs with the development of a small pre-defined electronic plate located in the bottom part of the orthosis. The temperature and heart rate are constantly monitored and displayed wirelessly on a smartphone. The target group are the patients active in sports or patients with minor injuries. Moreover, the orthosis can be used for body temperature and heart function monitoring during recovery period.
\end{abstract}

Keywords: orthosis design; smart orthosis; wrist orthosis; 3D print

\section{INTRODUCTION}

The work-related injuries of joints and hands represent one of the most common causes of immobilisation of hands and wrists. Studies [1] indicate a very significant amount in medical costs and a high degree of the absence of workers from work. At the same time the authors state that on the average $20-27 \%$ of all injuries in sport are the injuries of hands and joints, especially regarding sports that rely on more intensive use of the hands during physical activity [2]. Immobilisation as one of the ways to treat wrist injuries is a topic dealt with by many authors. By implementing digitalisation and additive technologies Koutny et al. [3] dealt with the possibility and methods of producing CAD models and customised orthoses. Apart from technology recommendation, the authors also give the solutions for a device which can be used for body parts digitalization and CAD models processing. In the development of customised orthoses, some of the authors [4-7] made tests with additive technologies with the aim of merging digitalised model for every patient individually. With the development of additive technologies, J. Li and H. Tanaka [4] showed the impacts of manufacturing time and time shares of pre-preparation and production of single orthosis. Additionally, the impact of modelling skill and individual model production considering the weight reduction and breathability of the orthosis is studied. Koutny et al. [3] compared the scanning devices as the basis for developing credible models of body extremities to make the orthoses and prosthetic aids. Fromme et al. [8] focused on the design of orthosis made of light materials such as fabrics and fixators with air in the form of muscles wrapped in fabric and adhesive Velcro strips. By changing the pressure inside the air pocket, the suspension of the joint and a restriction in the joint movement are allowed. A parallel branch of rehabilitation in orthopaedics relies on the application of sensors in medicine. A review by Reinkensmeyer [9] indicated an increasing trend in the implementation of sensors and active measuring devices in real time over the last fifteen years. It indicates the possibility of connecting wireless protocols in connecting the sensors to mobile devices. Avila et al. [10] presented a development concept of a device that can activate individual muscles for the rehabilitation purpose aimed for home use. Another possible application of sensors and devices can be found in the rehabilitation of the patient after heart attack. Toth et al. [11] described the derived conceptual design of the orthoses with actuators and their application in rehabilitation of the patients after a heart attack.

Jovanov et al. [12] provided a concept of wireless network for multiple devices that can communicate through a joint platform in a constant body monitoring. The authors state a possibility of centrally connecting several sensors all over the body in a single common platform. Such approach allows active monitoring of the body condition with a possibility of real-time monitoring if there is a feedback to the person who is supervising the patient.

The concept of smart orthosis device is aimed towards standardizing the parts of the orthosis through combining adaptive parts into a whole resulting in a predefined final product for the user. The combination of sizes and forms makes it possible to simplify the long process of digitalizing and modelling patient's forearm along with reducing the production costs. Another function of a smart orthosis is the daily monitoring of the patient's condition using basic temperature and heart rate sensors aiming at early diagnosis and active monitoring of the patient's condition.

\section{MATERIALS AND METHODS}

General approach to orthosis design is based on using available tools and optimisation methods which can be applied to develop personalised devices for every individual patient. In order to use the already pre-prepared models of the patients' forearms the 3D CAD software Inventor is used to create the $3 \mathrm{D}$ architecture of the orthosis model. The baseline for the basic model was the average adult male, aged 26 to 35 , of average height and size. Although arm and body models are not generally strictly standardised, a variation with respect to age and height can be noted. Due to a large number of models and variations, a standardisation model according to the $50^{\text {th }}$ percentile is proposed, and ready-made models of the body and forearm shape are taken as the basis 
for prototype dimensions of the orthosis shape. In this case the orthosis is designed for the left arm only. According to the adopted model the selected version of the product is divided longitudinally in two parts (Fig. 1). This ensures relatively easier installation on the injured forearm in the event of immobilization. Other methods of orthosis modelling were considered which include putting the orthosis on in the form of a glove or the minimalist approach with flat surfaces. However, due to the problem of implementing sensors and the danger of relatively poor contact of the sensor with the hand, a poorer signal transmission from skin to the sensors can occur in abovementioned cases.

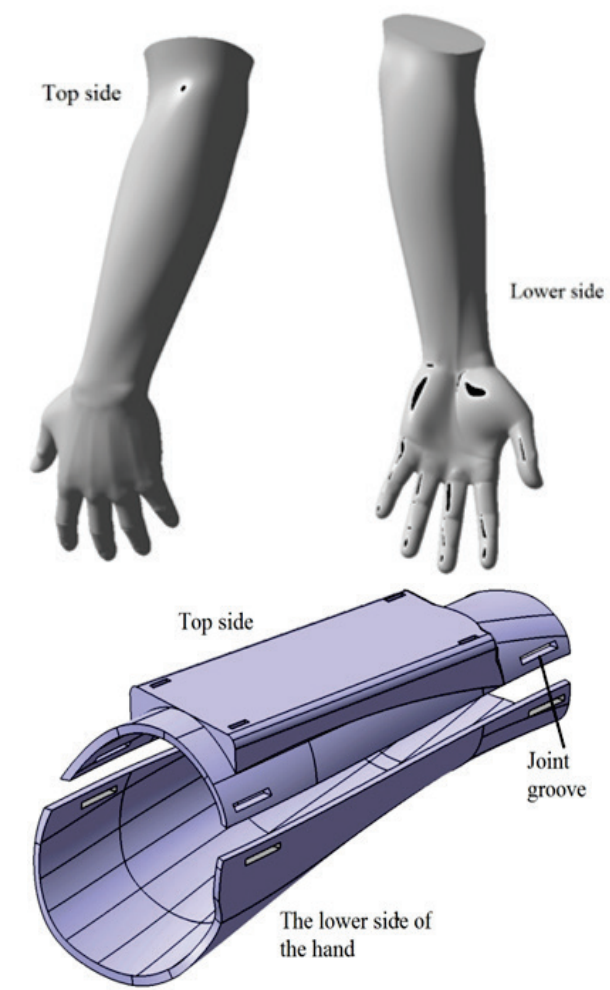

Figure $13 \mathrm{D}$ model of the arm and initial 3D model of the orthosis cut longitudinally

Modularity is ensured in a such way that the upper part of the orthosis is at the same time the central part on which other elements of the system are connected. Each part can be produced in a different size thus ensuring modularity based on the size of the human forearm. The part production is carried out by means of multi-material 3D printing on commercially available 3D printer model Prusa i3 MK3S. The materials used were PLA and 5G. The performed design indicates a satisfactory degree of accuracy, very low mass whereas the stiffness of the parts is obtained by applying curved surfaces. The connection of the upper and lower part of the orthosis is achieved by connecting grooves and application of flexible materials and self-adhesive Velcro strips.

The fixation of the wrist is the basic orthopaedic function of the presented orthosis. The fixation is ensured through a profiled groove and separate three inserts $-\mathrm{Z}$ bracket (Fig. 2 ), which is connected to the fingers by means of an elastic band. After design iteration, the angle of $15^{\circ}$ is employed ensuring optimal position of the hand which maximally relaxes the fixated joint.
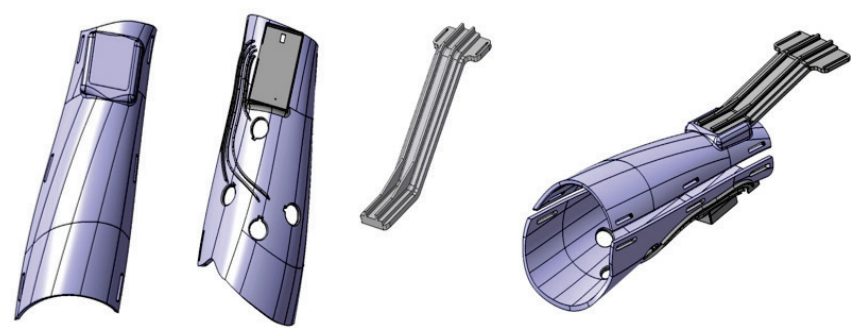

Figure 2 Basic parts of the orthosis (final model) and Z bracket fixation

The lower part of the orthosis (Fig. 2) is used for installation of sensors, wires and PCB electronics. Integrated PCB electronics controls the entire system and transmits the signal to the mobile device or computer wirelessly. To determine the optimal measurement points for temperature sensor locations, infrared recording of the temperature field of the patient's arm is performed. An infrared camera model BOSCH GTC $400 \mathrm{C}$ was used for the recording, with emissivity factor $\mathcal{E}=0.98$ according to [13]. The presented results of the forearm area (Fig. 3) show that there is no significant change in temperature over the surface of the hand. The temperature differences for the upper part of the forearm are within $2.1^{\circ} \mathrm{C}$ whereas the lower arm is in a total temperature difference of $1.3{ }^{\circ} \mathrm{C}$. Since there are no major differences in the temperature field on three respondents, it is concluded that the impact of the position is not a significant variable for the measurement of the body temperature in the forearm area. Four test points were selected for the implementation of the temperature sensors. The selected positions of the temperature sensors have been selected according to the positions on the hand that ensure the greatest possibility of good contact with the skin during use. The area around the wrist, due to the constant contact with the orthosis during tightening, is made solid with a through hole for the heart rate sensor placement.

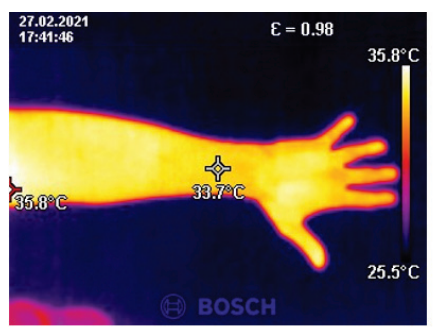

a)

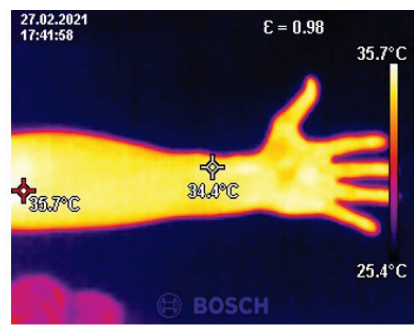

b)
Figure 3 Thermal image of the hand: a) Top side; b) Lower side

\subsection{Selection of Sensors and Components}

The measurement sensors used in the presented design:

- The temperature sensor DS1921H, Tab. 1, Fig. 4

- The heart rate sensor ROHM's BH1790GLC, Tab. 2, Fig. 5

- Bluetooth communication module RN42 
The temperature sensor DS1921H is a wide temperature range and long battery life sensor. Fit for moderately long logging rates, this simple sensor is perfect for any business looking to track and regulate temperature in their working environment. Applications for this sensor include tracking and regulating human or animal temperatures.

Table 1 Technical specification DS1921H

\begin{tabular}{|c|c|}
\hline Temperature range & $+15^{\circ} \mathrm{C}$ to $+46{ }^{\circ} \mathrm{C}$ \\
\hline Accuracy & $\pm 1{ }^{\circ} \mathrm{C}$ \\
\hline Resolution & $0.125^{\circ} \mathrm{C}$ \\
\hline Logging rate & $1-255 \mathrm{~min}$ \\
\hline
\end{tabular}

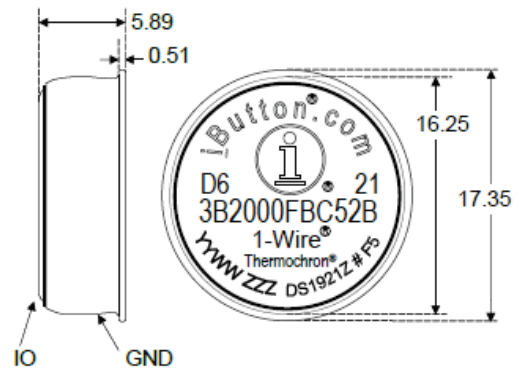

Figure $4 \mathrm{DS} 1921 \mathrm{H}$ temperature sensor - dimension

BH1790GLC is optical sensor for heart rate monitoring in which LED driver and green light detection photodiode are incorporated. This device drives LED and provides the intensity of light reflected from the body. LED brightness can be adjusted by LED driver current and light emitting period. The photodiode having the high sensitivity for green light, excellent wave-length selectivity and excellent circuit characteristics results in accurate pulse wave detection.

Table 2 Technical specification BH1790GLC

\begin{tabular}{|c|c|}
\hline VCC1 Voltage Range & $2.5 \mathrm{~V}$ to 3.6 V \\
\hline VCC2 Voltage Range & $1.7 \mathrm{~V}$ to 3.6 V \\
\hline Current Consumption & $200 \mu \mathrm{A}$ (Typ) \\
\hline Standby Mode Current & $0.8 \mu \mathrm{A}$ (Typ) \\
\hline Operating Temperature Range & $-20^{\circ} \mathrm{C}$ to $+85^{\circ} \mathrm{C}$ \\
\hline
\end{tabular}

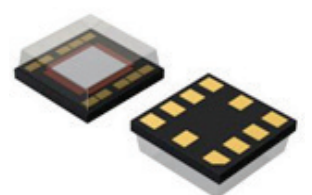

Figure 5 BH1790GLC optical sensor

The project defines the significance of interactive orthosis and for this purpose a special PCB electronic circuit is developed, which connects several sensors and transfers signals from the measuring points to a computer wirelessly. Due to the need for a compact size and robustness, it is decided to place the heart rate sensor as close to the skin as possible. The electronic circuit according to Fig. 6 shows the connected modules around the ATmega 644 microcontroller. The electronics has been designed for full mobility and independence: it contains an independent source of power by a separate battery and acts as an independent device that transmits data to the paired device. During the design phase, special attention is placed on the size of the electronic assembly. A design requirement was that maximum allowable dimensions of the electronics should not exceed half width of the orthosis in the wrist area. The electronics concept is based as a universal module with four inputs for temperature measurement and one sensor for heart rate measurement. It is possible to expand the number of sensors that could provide more information about the patient's condition, such as a pressure sensor or a sweat analyser. This would open the possibility of further expansion of the application. The presented PCB concept is only the basic idea of a central system of data collection which provides information to a mobile device where post-processing and data analysis are performed.
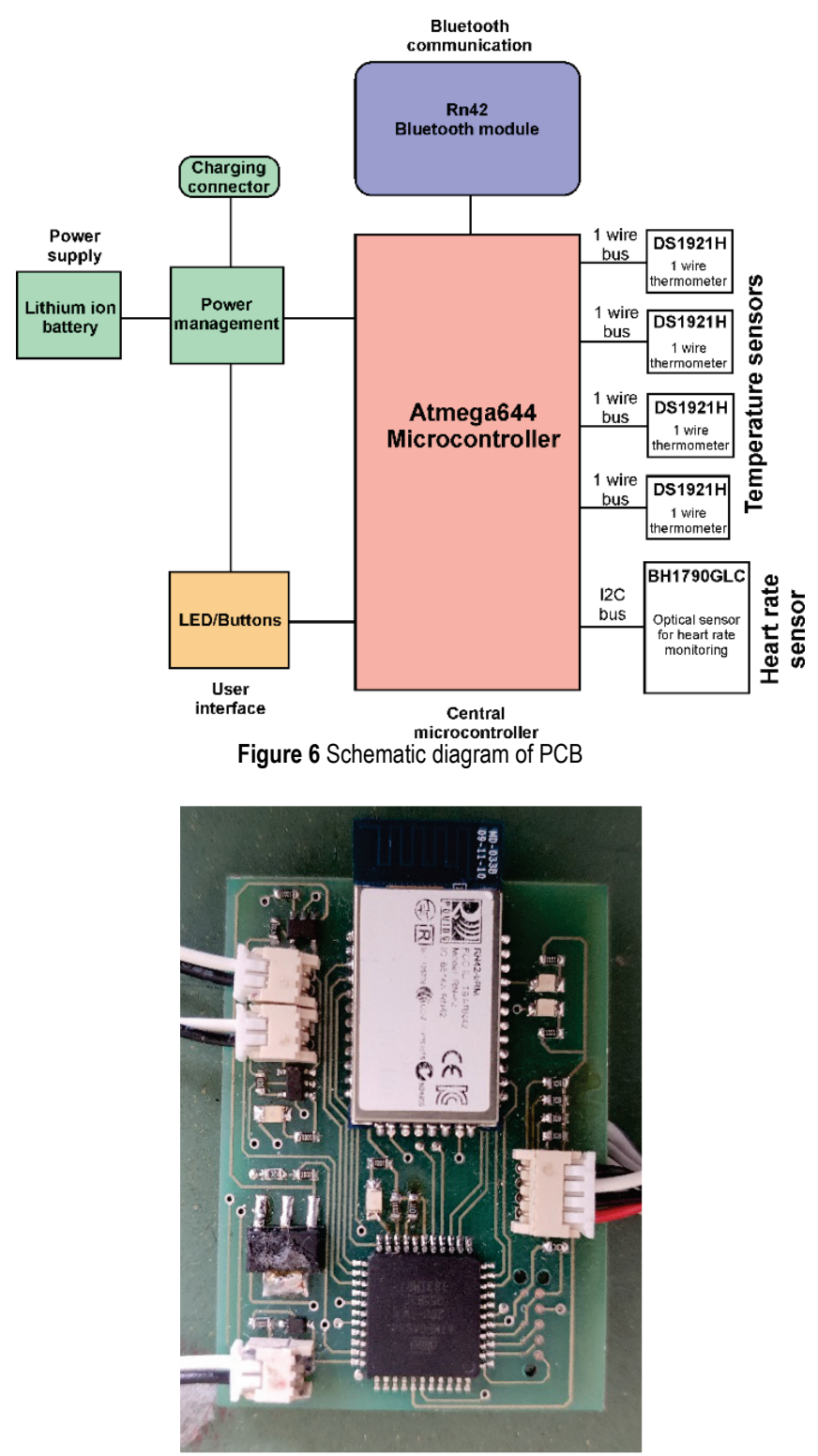

Figure 7 Produced PCB sample

\section{TEST OPERATION AND RESULTS}

PCB, as a central unit, is located on the lower part of the orthosis (Fig. 8). Fixator is placed within the protected housing and fixed together with the battery to the housing. The lower part's thickness, i.e. the distance between the 
heart rate sensor and the skin, amounts to $1.5 \mathrm{~mm}$. Tests have shown that with changing the distance it is necessary to adjust the signal strength and to regulate the sensor sensitivity.

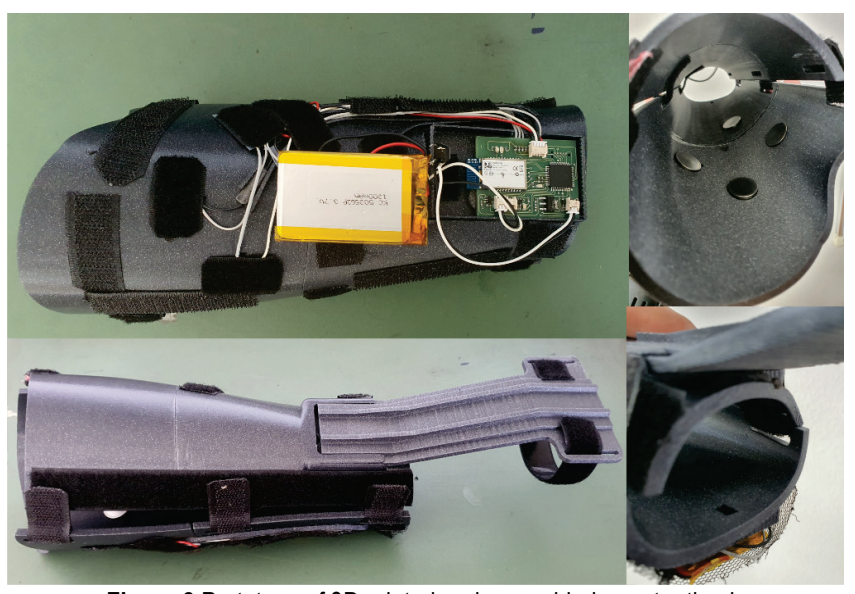

Figure 8 Prototype of 3D printed and assembled smart orthosis

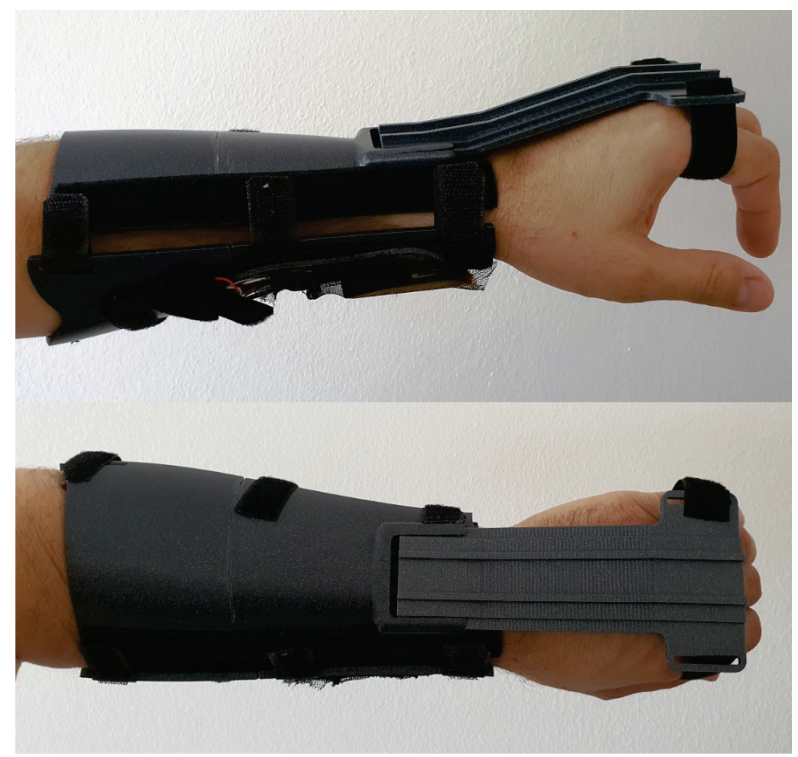

Figure 9 Prototype of smart orthosis in test operation

The test operation and the proof of concept start by connecting electronics with 3D printed mechanical parts (Fig. 9). By turning on the electronics the sensor readings start and the rise of temperatures at four measuring points with predicted converge towards normal human body can be observed. Individual deviations of the measuring point do not indicate significant differences according to the diagram in Fig. 10. At the same time, the diagram in Fig. 11 shows that the heart rate measurement does not provide expected heart rates. Average display of the normal resting heart rate for a human body ranges between 60 and 100 beats per minute. In the case of physical activity, the heart rate rises to higher values. The test measurements showed that the average heart rate ranges from 50 to -5 , and the effect of light penetration to the photosensitive sensor was observed. This confirmed that perhaps the position of the heart rate sensor is not optimal. Based on the observations, the proposal is to create a separate PCB for patient's heart rate measurement only.
Apart from unequal distance, the possible problem may be inappropriate point of measurement, although it has been proven that the place on the wrist can be used as one of the measuring points.

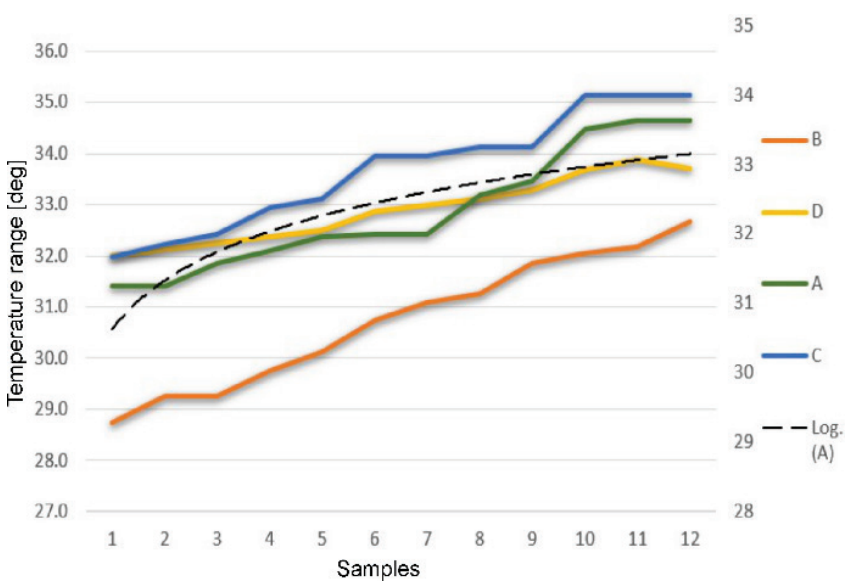

Figure 10 Temperature changing in time on 4 measuring sensors $(A, B, C, D)$, log A -temperature tendency changing

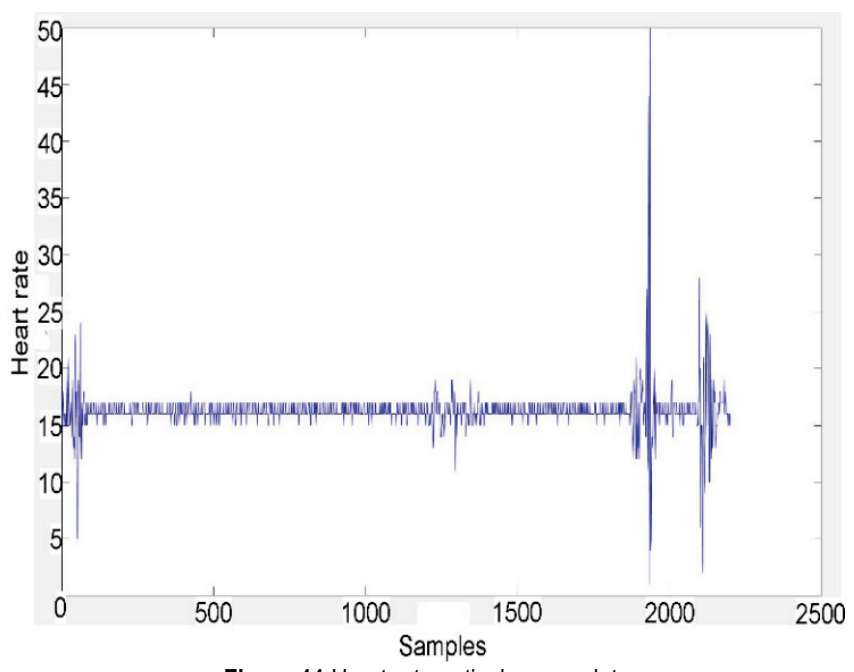

Figure 11 Heart rate optical sensor data

\section{CONCLUSIONS}

In the initial phase of the project, the possibility of measuring the temperature by skin contact was analysed based on the assumption that the places on the forearm with the highest blood flow and the thinnest skin provide the most reliable measuring points. According to the measurements, it was found that the lower part of the forearm is a slightly better variant than the upper part. The reason may be the thinner skin and less hair coverage. From the design point of view, the placement of electronics in the lower part of the orthosis is more suitable due to the proximity of the PCB and compactness of the design. The choice of orthosis production method by applying additive technology satisfies the moderate use rate. With the proposed shape of the orthosis it is possible to achieve sufficient rigidity of the structure which ensures joint fixation. This has been achieved by producing a $\mathrm{Z}$ bracket which is connected over a quick connection to the profiled groove in the upper part of the orthosis. Connecting the fingers to the $\mathrm{Z}$ bracket via the Velcro strip and the 
bandage allows the joint to be fixed in the position which maximally relieves it and allows recovery. The suggested structure of the PCB allows compact design that can be worn on the arm without interference during use. The distribution of the temperature sensors performed according to the area of the highest temperature differences on the skin surface by measuring, shows a correlation on four measuring points and a small influence of the choice of the measuring point on the measurement in the area of the lower part of the forearm. The pulse measurement by photosensitive sensors is commonly employed method of determining pulse. The presented design with integrated heart rate sensor in the PCB body indicates a possible discontinuity of measurement and greater scatter in the collected data. Eventually, during use, this could lead to unreliable results and it is recommended to exclude the sensor from the basic electronics and to create a module that would have a better and more constant contact with the skin. The used measuring point can be considered reliable for the tested type of measurement.

In future considerations, the changes that should be implemented in order to improve the presented concept of smart orthosis refer to the shape optimisation in order to reduce the contact surfaces in the upper part of the orthosis and standardisation of the joints to conform to different forearm sizes. Considering the presented concept in the terms of a measurement system, the size and response time of temperature sensors could be reduced by implementing more optimized sensors. Moreover, the separation of the heart rate sensor into a separate module and integration with the housing should be a priority. The control system and mobile application provide the possibility of optimisation and addition of other types of sensors into the presented concept, such as sweat sensors and pressure sensors.

\section{Notice}

The paper was presented at MOTSP $2021-12^{\text {th }}$ International Conference Management of Technology - Step to Sustainable Production, which took place in Poreč/Porenzo, Istria (Croatia), on September 8-10, 2021. The paper will not be published anywhere else.

\section{REFERENCES}

[1] Barr, A. E., Barbe, M. F., \& Clark, B. D. (2004). Work-Related Musculoskeletal Disorders of the Hand and Wrist. Epidemiology, Pathophysiology, and Sensorimotor Changes, 34(10), 610-627. https://doi.org/10.2519/jospt.2004.34.10.610

[2] Heath, L. (2010). Wrist and hand injuries in sport, Wrist and Hand Injuries in Sport. In book: Sports Rehabilitation and Injury Prevention, Publisher: John Wiley \& Sons, Ltd. January 2010. https://doi.org/10.1002/9781118685150.ch19

[3] Koutny, D., Palousek, D., Koutecky, T., Zatocilova, A., Rosicky, J., \& Janda, M. (2012). 3D digitalization of the human body for use in orthotics and prosthetics. World Academy of Science, Engineering and Technology; 6. https://doi.org/10.5281/zenodo.1331055

[4] Li, J. \& Tanaka H. (2018). Feasibility study applying a parametric model as the design generator for $3 \mathrm{D}$ - printed orthosis for fracture immobilization. 3D Printing in Medicine, 4(1). https://doi.org/10.1186/s41205-017-0024-1
[5] Banga, H. K., Kalra, P., Belokar, R. M., \& Kumar, R. (2020). Design and Fabrication of Prosthetic and Orthotic Product by 3D Printing. In book: Orthotics and Prosthetics. https://doi.org/10.5772/intechopen.94846

[6] Fernandez-Vicente, M., Chust A. E., \& Conejero, A. (2017). Low Cost Digital Fabrication Approach for Thumb Orthoses. Rapid Prototyping Journal, 23(6). https://doi.org/10.1108/RPJ-12-2015-0187

[7] Mohammed, M. I. \& Fay, P. (2018). Design and additive manufacturing of a patient specific polymer thumb splint concept. Solid Freeform Fabrication 2018: Proceedings of the $29^{\text {th }}$ Annual International; Solid Freeform Fabrication Symposium - An Additive Manufacturing Conference, Reviewed Paper.

[8] Fromme, N. P., Camenzind, M., Riener, R., \& Rossi R. M. (2020). Design of a lightweight passive orthosis for tremor suppression. Journal of NeuroEngineering and Rehabilitation, 17. https://doi.org/10.1186/s12984-020-00673-7

[9] Reinkensmeyer, D. J. (2019). JNER at 15 years: analysis of the state of neuroengineering and rehabilitation. Journal of NeuroEngineering and Rehabilitation, 16. https://doi.org./10.1186/s12984-019-0610-0

[10] Romero Avila, E., Junker, E., \& Disselhorst-Klug, C. (2020). Introduction of a sEMG Sensor System for Autonomous Use by Inexperienced Users. Sensors, 20(24). https://doi.org/10.3390/s20247348

[11] Toth, L., Schiffer, A., Nyitrai, M., Pentek, A., Told, R., \& Maroti, P. (2020). Developing an anti-spastic orthosis for daily home-use of stroke patients using smart memory alloys and 3D printing technologies. Materials \& Design, 195. https://doi.org/10.1016/j.matdes.2020.109029

[12] Jovanov, E., Milenkovic, A., Otto, C., \& and de Groen, P. C. (2005). A wireless body area network of intelligent motion sensors for computer assisted physical rehabilitation. Journal of NeuroEngineering and Rehabilitation, 2. https://doi.org/10.1186/1743-0003-2-6

[13] Bernard, V., Staffa, E., Mornstein, V., \& Bourek, A. (2012). Infrared camera assessment of skin surface temperature-Effect of emissivity. Physia Medica, 29(6), 583-591 https://doi.org/10.1016/j.ejmp.2012.09.003

\section{Authors' contacts:}

Zoran Domitran, Assistant Professor, PhD

(Corresponding author)

Faculty of Mechanical Engineering and Naval Architecture,

University of Zagreb,

Ivana Lučića 5, 10000 Zagreb, Croatia

zoran.domitran@fsb.hr

Robert Mašović, Research Assistant

Faculty of Mechanical Engineering and Naval Architecture,

University of Zagreb,

Ivana Lučića 5, 10000 Zagreb, Croatia

robert.masovic@fsb.hr

Jure Serdar, Research Assistant

University Hospital Centre Zagreb,

Kišpatićeva 12, 10000 Zagreb, Croatia

ortopedija@kbc-zagreb.com

Mislav Jelić, Associate Professor, PhD University Hospital Centre Zagreb, Kišpatićeva 12, 10000 Zagreb, Croatia ortopedija@kbc-zagreb.com 